\title{
Bosonization within thermofield dynamics approach
}

\author{
R. L. P. G. Amaral* \\ Instituto de Física \\ Universidade Federal Fluminense
}

Av. Litorânea S/N, Boa Viagem, Niterói, CEP. 24210-340

Rio de Janeiro - Brasil

E-mail: rubens@if.uff.br

\section{V. Belvedere}

Instituto de Física

Universidade Federal Fluminense

Av. Litorânea S/N, Boa Viagem, Niterói, CEP. 24210-340

Rio de Janeiro - Brasil

\section{K. D. Rothe}

Institut für Theoretische Physik

Universität Heidelberg

Philosophenweg 16, D-69120 Heidelberg

Germany

\begin{abstract}
We present an operator realization for the bosonization of fermions in $1+1$ dimensions, at finite, non-zero temperature $T$. This is achieved in the framework of the real time formalism of Thermofield Dynamics. The well known two-dimensional Fermion-Boson correspondences at zero temperature are shown to hold also at finite temperature. In order to emphasize the usefulness of the operator realization for handling a large class of two-dimensional quantum field-theoretic problems, we contrast this global approach with the cumbersome calculation of the fermioncurrent two-point function in the imaginary-time formalism and real time formalisms.
\end{abstract}

Fifth International Conference on Mathematical Methods in Physics - IC2006

April 24-28 2006

Centro Brasilerio de Pesquisas Fisicas, Rio de Janeiro, Brazil

${ }^{*}$ Speaker. 


\section{Introduction}

The bosonization of fermions has proved in the past to be a very useful technique for solving quantum field theoretic models in 1+1 dimensions [1]. Recently this Fermion-Boson mapping has been discussed for the case of a finite Temperature, using the imaginary time formalism [2] and real time formalism[3].

The following mapping at zero temperature in $(1+1) \mathrm{D}$ is well known:

$$
\begin{gathered}
\bar{\psi} i \gamma^{\mu} \partial_{\mu} \psi \rightarrow \frac{1}{2}: \partial_{\mu} \phi \partial^{\mu} \phi:, \\
\bar{\psi} \gamma^{\mu} \psi \rightarrow-\frac{1}{\sqrt{\pi}} \varepsilon^{\mu \nu} \partial_{\nu} \phi, \\
M \bar{\psi} \psi \rightarrow-\frac{\mu M}{\pi}: \cos 2 \sqrt{\pi} \phi: .
\end{gathered}
$$

Here we establish this mapping at finite temperature through operatorial methods within thermofield dynamics formalism $[4,5,6]$. We also contrast this approach with direct computations of fermion-current two-point function in imaginary-time and real-time computations. The explicit expression for the fermion field in terms of the massless scalar field is provided. This work condensates the presentation provided in [7].

\section{The Transmutation of Fermi-Dirac to Bose-Einstein Statistics}

We illustrate here how the statistical transmutation, Fermi-Dirac $==>$ Bose-Einstein, occurs in imaginary and real time formalisms.

\subsection{Imaginary time}

The current 2-point function in the imaginary time formalism is well known. Here we work with light-cone coordinates $x_{ \pm}=x_{1} \pm i x_{2}$ and $j_{ \pm}=j_{1} \pm i j_{2}$, so that the Euclidean two-point current correlator at zero temperature is given by

$$
\begin{aligned}
<j_{+}(x) j_{+}(y)> & =-\operatorname{tr}\left(\gamma_{+} i S_{E}(x-y) \gamma_{+} i S_{E}(y-x)\right) \\
& =\int \frac{d^{2} k}{(2 \pi)^{2}} \Pi_{++}^{(E)}(k) e^{i k \cdot(x-y)}
\end{aligned}
$$

with,

$$
\Pi_{++}^{(E)}(k)=4 \int \frac{d^{2} p}{(2 \pi)^{2}}\left[(p-k)_{1}+i(p-k)_{2}\right]\left[p_{1}+i p_{2}\right] D_{E}(p-k) D_{E}(p)
$$

and

$$
D_{E}(q)=\frac{1}{q^{2}}=\frac{1}{\left(q_{1}+i q_{2}\right)\left(q_{1}-i q_{2}\right)}
$$




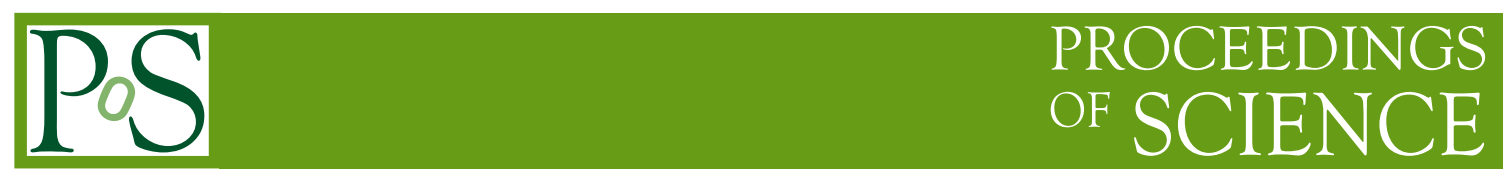

At Finite temperature and within imaginary time formalism it becomes expressed as

$$
<j_{+}(x) j_{+}(y)>_{\beta}=\sum_{\ell} \frac{1}{\beta} \int \frac{d k_{1}}{2 \pi} e^{i k_{1}\left(x_{1}-y_{1}\right)} e^{i \omega_{\ell}\left(x_{2}-y_{2}\right)} \tilde{\Pi}_{++}^{(E)}\left(k_{1}, \omega_{\ell}\right),
$$

where $\omega_{\ell}=\frac{2 \pi \ell}{\beta}=$ are the Matsubara frequencies.

$$
\tilde{\Pi}_{++}^{(E)}\left(k_{1}, \omega_{\ell}\right)=4 \sum_{m} \frac{1}{\beta} \int \frac{d p_{1}}{2 \pi} \frac{1}{\left[p_{1}-i\left(m+\frac{1}{2}\right) \frac{2 \pi}{\beta}\right]\left[p_{1}-k_{1}-i\left(m-\ell+\frac{1}{2}\right) \frac{2 \pi}{\beta}\right]} .
$$

It is important to notice the lack of uniform convergence above so that the order in which the sum and integral are to be performed are not interchangeable.

Indeed if we first sum by using that,

$$
\sum_{m} \frac{1}{[(m+(1 / 2)+i x]} \frac{1}{[(m+(1 / 2)+i y]}=\frac{\pi}{x-y}(\tanh \pi x-\tanh \pi y),
$$

it results

$$
\tilde{\Pi}_{++}^{(E)}\left(k_{1}, \omega_{\ell}\right)=-\frac{2}{k_{1}-i \frac{2 \pi \ell}{\beta}} \int \frac{d p_{1}}{2 \pi}\left(\tanh \frac{\beta}{2} p_{1}-\tanh \frac{\beta}{2}\left(p_{1}-k_{1}\right)\right)
$$

with

$$
\tanh \frac{\beta}{2} q_{1}=\varepsilon\left(q_{1}\right)\left(1-2 N_{F}\left(\left|q_{1}\right|\right)\right)
$$

where $N_{F}\left(\left|q_{1}\right|\right)$ is the Fermi-Dirac distribution.

$$
N_{F}\left(\left|q_{1}\right|\right)=\frac{1}{e^{\beta\left|q_{1}\right|}+1} .
$$

The integration over $p_{1}$ results now in:

$$
\tilde{\Pi}_{++}^{(E)}\left(k_{1}, \omega_{\ell}\right)=-\frac{2}{\pi} \frac{k_{1}}{k_{1}-i \frac{2 \pi \ell}{\beta}} .
$$

On the other hand if we first integrate then sum we notice that the $p_{1}$ integral leads to :

$$
\begin{aligned}
& \int \frac{d p_{1}}{2 \pi} \frac{1}{\left[p_{1}-i(m+1 / 2) \frac{2 \pi}{\beta}\right]\left[p_{1}-k_{1}-i(m-\ell+1 / 2) \frac{2 \pi}{\beta}\right]}= \\
& =-i[\theta(2 m+1) \theta(-2 m+2 \ell-1)-\theta(-2 m-1) \theta(2 m-2 \ell+1)] \frac{1}{k_{1}-i \frac{2 \pi \ell}{\beta}}
\end{aligned}
$$

Now the sum over $m$ results in 


$$
\begin{aligned}
\tilde{\Pi}_{++}^{(E)}\left(k_{1}, \omega_{\ell}\right) & =\frac{-4 i}{\beta}(\theta(\ell)+\theta(-\ell-1)) \frac{\ell}{k_{1}-i \frac{2 \pi \ell}{\beta}} \\
& =-\frac{2}{\pi} \frac{i \frac{2 \pi \ell}{\beta}}{k_{1}-i \frac{2 \pi \ell}{\beta}} .
\end{aligned}
$$

This is in contrast with the previous computation. In order to fix the ambiguity we take the mean of the two results

$$
\tilde{\Pi}_{++}\left(k_{1}, \omega_{\ell}\right)=-\frac{1}{\pi} \frac{k_{1}+i \frac{2 \pi \ell}{\beta}}{k_{1}-i \frac{2 \pi \ell}{\beta}} .
$$

With this we preserve Lorentz invariance in the zero temperature limit. We further obtain that:

$$
<j_{+}(x) j_{+}(y)>_{\beta}=-\frac{1}{\pi} \partial_{+}^{2} D_{E}^{(\beta)}(x-y)
$$

where

$$
D_{E}^{(\beta)}(z)=\sum_{\ell} \frac{1}{\beta} \int \frac{d k_{1}}{2 \pi} \frac{e^{i k_{1} z_{1}} e^{i \frac{2 \pi \ell}{\beta} z_{2}}}{\left(k_{1}^{2}+\frac{2 \pi \ell}{\beta}\right)^{2}}
$$

The final sum leads to

$$
D_{E}^{(\beta)}(z)=\int \frac{d^{2} k}{(2 \pi)^{2}} e^{i k \cdot z}\left[\frac{1}{k^{2}}-i 2 \pi \delta\left(k^{2}\right) N_{B}\left(\left|k_{1}\right|\right)\right]
$$

where $N_{B}\left(\left|k_{1}\right|\right)$ is the Bose-Einstein distribution

$$
N_{B}\left(\left|k_{1}\right|\right)=\frac{1}{e^{\beta\left|k_{1}\right|}-1}
$$

By rotating to Minkovski space

$$
D_{E}^{(\beta)}(z) \rightarrow i D_{B}^{(\beta)}(z)=i \int \frac{d^{2} k}{(2 \pi)^{2}} e^{-i k \cdot z}\left[\frac{1}{k^{2}+i \varepsilon}+2 \pi i \delta\left(k^{2}\right) N_{B}\left(\left|k_{1}\right|\right)\right]
$$

So that:

$$
<T j_{+}(x) j_{+}(y)>_{\beta}=\frac{1}{\pi} \partial_{+}^{2} i D_{B}^{(\beta)}(x-y) .
$$

This result agrees with the identification

$$
\bar{\psi} \gamma^{\mu} \psi \rightarrow-\frac{1}{\sqrt{\pi}} \varepsilon^{\mu v} \partial_{v} \phi
$$




\subsection{Realtime}

The current 2-point function in the real time formalism is given by

$$
\Pi_{++}(k)=4 \int \frac{d^{2} p}{(2 \pi)^{2}}(p-k)_{+} p_{+} D_{F}^{(\beta)}(p-k) D_{F}^{(\beta)}(p),
$$

with

$$
D_{F}^{(\beta)}(q)=\frac{1}{q^{2}+i \varepsilon}+2 \pi i \delta\left(q^{2}\right) N_{F}(|\vec{q}|) .
$$

where the Fermi-Dirac statistical factor is

$$
N_{F}\left(\left|q_{1}\right|\right)=\frac{1}{e^{\beta\left|q_{1}\right|}+1} .
$$

The double integral is performed with: 1) In the temperature independent terms we require $\left.\partial_{\mu} J^{\mu}=0.2\right)$ In the temperature dependent terms use repeatedly the decomposition

$$
\frac{\alpha}{\alpha(x)+\imath \varepsilon}=\mathscr{P}\left(\frac{1}{x}\right)-\imath \Pi \varepsilon(\alpha) \delta(x) .
$$

This leads to

$$
<T j_{\mu}(x) j_{v}(y)>_{\beta}=\frac{1}{\pi} \tilde{\partial}_{\mu} \tilde{\partial}_{v} i D_{B}^{(\beta)}(x-y)
$$

where

$$
D_{B}^{(\beta)}(k)=\left(\frac{1}{k^{2}+i \varepsilon}+2 \pi i \delta\left(k^{2}\right) N_{B}\left(\left|k_{1}\right|\right)\right)
$$

The conclusion is that the results are necessary to establish the finite temperature bosonization. But it becomes clear that this procedure is not very illuminating with regards to showing how the bosonization machinery works.

\section{Free Massless Scalar thermofield}

As a first step to two-dimensional bosonization we review the quantization of the massless scalar $\phi$. This will be done within the Thermofield dynamics approach: we double the number of fields by introducing a fictitious system with field $\widetilde{\phi}$ besides the physical field $\phi$.

$$
\mathscr{L}_{T}=\mathscr{L}-\widetilde{\mathscr{L}}=\frac{1}{2} \partial_{\mu} \phi \partial^{\mu} \phi-\frac{1}{2} \partial_{\mu} \widetilde{\phi} \partial^{\mu} \widetilde{\phi}
$$

As an start point to quantization we consider the creation and annihilation operators for the chiral field components:

$$
\begin{aligned}
& \phi\left(x^{ \pm}\right)=\int_{0}^{\infty} \frac{d p^{1}}{\sqrt{4 \pi\left|p^{1}\right|}}\left[f_{p}\left(x^{ \pm}\right)\left(\begin{array}{c}
a\left(-p^{1}\right) \\
a\left(p^{1}\right)
\end{array}\right)+f_{p}^{*}\left(x^{ \pm}\right)\left(\begin{array}{c}
a^{\dagger}\left(-p^{1}\right) \\
a^{\dagger}\left(p^{1}\right)
\end{array}\right)\right], \\
& \widetilde{\phi}\left(x^{ \pm}\right)=\int_{0}^{\infty} \frac{d p^{1}}{\sqrt{4 \pi\left|p^{1}\right|}}\left[f_{p}^{*}\left(x^{ \pm}\right)\left(\begin{array}{c}
\widetilde{a}\left(-p^{1}\right) \\
\widetilde{a}\left(p^{1}\right)
\end{array}\right)+f_{p}\left(x^{ \pm}\right)\left(\begin{array}{c}
\widetilde{a}^{\dagger}\left(-p^{1}\right) \\
\widetilde{a}^{\dagger}\left(p^{1}\right)
\end{array}\right)\right],
\end{aligned}
$$


with

$$
f_{p}(x)=e^{-i p^{\mu} x_{\mu}}
$$

We define the thermal vacuum by

$$
|0(\beta)\rangle=U_{B}\left(\theta_{B}\right)|0, \widetilde{0}\rangle
$$

with the unitary operator $U_{B}\left(\theta_{B}\right)$

$$
U_{B}\left(\theta_{B}\right)=e^{-i \mathscr{Q}\left(\theta_{B}\right)}=e^{-\int_{-\infty}^{+\infty} d p^{1}\left(\widetilde{a}\left(p^{1}\right) a\left(p^{1}\right)-a^{\dagger}\left(p^{1}\right) \widetilde{a}^{\dagger}\left(p^{1}\right)\right) \theta_{B}\left(\left|p^{1}\right|, \beta\right)},
$$

where the Bogoliubov parameter $\theta_{B}\left(\left|p^{1}\right|, \beta\right)$ is:

$$
\sinh \theta_{B}\left(\left|p^{1}\right| ; \beta\right)=\frac{e^{-\beta\left|p^{1}\right| / 2}}{\sqrt{1-e^{-\beta\left|p^{1}\right|}}}
$$

which is related to the Bose-Einstein distribution

$$
N_{B}\left(\left|p^{1}\right| ; \beta\right)=\sinh ^{2} \theta_{B}\left(\left|p^{1}\right| ; \beta\right)=\frac{1}{e^{\beta\left|p^{1}\right|}-1} .
$$

The thermal operators can now be introduced as

$$
a\left(p^{1} ; \beta\right)=U_{B}\left(-\theta_{B}\right) a\left(p^{1}\right) U_{B}\left(\theta_{B}\right) .
$$

The thermal chiral fields $\phi\left(x^{ \pm} ; \beta\right)$ are obtained thereupon

With this the chiral components two-point functions are readily obtained

$$
\begin{aligned}
\left\langle 0, \widetilde{0}\left|\phi\left(x^{ \pm} ; \beta\right) \phi\left(y^{ \pm} ; \beta\right)\right| \widetilde{0}, 0\right\rangle= & D_{o}^{(+)}\left(x^{ \pm}-y^{ \pm}\right)+ \\
& +\frac{1}{2 \pi} \int_{0}^{\infty} \frac{d p}{p}\left[\cos p\left(x^{ \pm}-y^{ \pm}\right)\right] N_{B}(p ; \beta),
\end{aligned}
$$

where the first term is the zero temperature result. The integrals are performed by introducing independent infrared regularization parameters $\mu$ (zero temperature) and $\mu^{\prime}$ (temperature dependent term). It results

$$
\begin{aligned}
\left\langle 0, \widetilde{0}\left|\phi\left(x^{ \pm} ; \beta\right) \phi\left(y^{ \pm} ; \beta\right)\right| \widetilde{0}, 0\right\rangle= & -\frac{1}{4 \pi} \ln \left\{i \mu \frac{\beta}{\pi} \sinh \frac{\pi\left(x^{ \pm}-y^{ \pm}-i \varepsilon\right)}{\beta}\right\}+ \\
& +\frac{1}{2 \pi} z\left(\beta, \mu^{\prime}\right)
\end{aligned}
$$

Here $z$ is a constant ( singular for $\mu^{\prime} \rightarrow 0$ ) that plays an important role in bosonization as will be seen shortly.

The thermalized tilded fields, $\widetilde{\phi}\left(x^{ \pm} ; \beta\right)$, are analogously defined.

All the two-point functions are obtained thereupon 


\section{Wick ordered exponential and selection rules}

The building blocks for Mandelstam expression are given in terms of the chiral field components by:

$$
W\left(x^{ \pm} ; \lambda\right)=: e^{i \lambda \phi\left(x^{ \pm}\right)}: \doteq e^{i \lambda \phi^{(-)}\left(x^{ \pm}\right)} e^{i \lambda \phi^{(+)}\left(x^{ \pm}\right)} .
$$

We should require that

$$
\langle 0|W(x ; \lambda)| 0\rangle=\langle\widetilde{0}|\widetilde{W}(x ; \lambda)| \widetilde{0}\rangle=1 .
$$

Using that

$$
\begin{aligned}
& U_{B}\left(\theta_{B}\right) \phi^{( \pm)}(x) U_{B}^{-1}\left(\theta_{B}\right)=\phi_{c}^{( \pm)}(x ; \beta)-\widetilde{\phi}_{s}^{(\mp)}(x ; \beta), \\
& U_{B}\left(\theta_{B}\right) \widetilde{\phi}^{(+)}(x) U_{B}^{-1}\left(\theta_{B}\right)=\widetilde{\phi}_{c}^{( \pm)}(x ; \beta)-\phi_{s}^{(\mp)}(x ; \beta) .
\end{aligned}
$$

We obtain

$$
\begin{gathered}
\langle 0(\beta)|W(x ; \lambda)| 0(\beta)\rangle=\langle 0, \widetilde{0}|W(x ; \beta, \lambda)| \widetilde{0}, 0\rangle=\mathscr{Z}\left(\beta, \mu^{\prime}, \lambda^{2}\right), \\
=\langle 0(\beta)|\widetilde{W}(x ; \lambda)| 0(\beta)\rangle
\end{gathered}
$$

Thus the constant $\mathscr{Z}\left(\beta, \mu^{\prime}, \lambda^{2}\right)=e^{-\frac{\lambda^{2}}{4 \pi} z\left(\beta, \mu^{\prime}\right)}$ is incorporated in the renormalized Wick ordered operators.

A direct computation of the Wick ordered exponentials leads, for instance, to

$$
\begin{aligned}
\left\langle 0(\beta)\left|\prod_{j=1}^{n} W\left(x_{j} ; \lambda_{j}\right)\right| 0(\beta)\right\rangle=e^{-\frac{1}{4 \pi} z\left(\beta, \mu^{\prime}\right)\left(\sum_{j=1}^{n} \lambda_{j}\right)^{2}} \times & \prod_{i<j}^{n}\left[i \mu \frac{\beta}{\pi} \sinh \frac{\pi\left(x_{i}-x_{j}-i \varepsilon\right)}{\beta}\right]^{\frac{\lambda_{i} \lambda_{j}}{4 \pi}} .
\end{aligned}
$$

Note that the zero temperature selection rule

$$
\sum_{i=1}^{n} \lambda_{i}=0
$$

Eliminates simultaneously the dependence on both infrared parameters, $\mu$ and $\mu^{\prime}$.

With this we achieve that the Wick ordered exponentials live on a positive norm Hilbert Space, provided they are associated to conserved charges. 


\section{Free Massless Fermi thermofield}

The doubling of fields reads now

$$
\mathscr{L}_{T}=i \bar{\Psi} \gamma^{\mu} \partial_{\mu} \Psi=\mathscr{L}-\widetilde{\mathscr{L}}=i \bar{\psi} \gamma_{\mu} \partial_{\mu} \psi-\left(-i \overline{\widetilde{\psi}} \gamma^{\mu} \partial_{\mu} \widetilde{\psi}\right)
$$

With

$$
\begin{aligned}
& \psi(x)=\left(\begin{array}{l}
\psi\left(x^{+}\right) \\
\psi\left(x^{-}\right)
\end{array}\right), \\
& \widetilde{\psi}(x)=\left(\begin{array}{l}
\widetilde{\psi}\left(x^{+}\right) \\
\widetilde{\psi}\left(x^{-}\right)
\end{array}\right),
\end{aligned}
$$

where

$$
\begin{aligned}
& \psi\left(x^{ \pm}\right)=\int_{0}^{\infty} \frac{d p}{\sqrt{2 \pi}}\left\{f_{p}\left(x^{ \pm}\right) b(\mp p)+f_{p}^{*}\left(x^{ \pm}\right) d^{\dagger}(\mp p)\right\}, \\
& \widetilde{\psi}\left(x^{ \pm}\right)=\int_{0}^{\infty} \frac{d p}{\sqrt{2 \pi}}\left\{f_{p}^{*}\left(x^{ \pm}\right) \widetilde{b}(\mp p)+f_{p}\left(x^{ \pm}\right)\right\} \widetilde{d}^{\dagger}(\mp p) .
\end{aligned}
$$

The unitary operator leading to the thermofields is now given by

$$
U_{F}\left(\theta_{F}\right)=e^{-\int_{-\infty}^{\infty} d p \theta_{F}\left(\left|p^{1}\right|, \beta\right)\left(\widetilde{b}\left(p^{1}\right) b\left(p^{1}\right)-b^{\dagger}\left(p^{1}\right) \widetilde{b}^{\dagger}\left(p^{1}\right)+\widetilde{d}\left(p^{1}\right) d(p)-d^{\dagger}\left(p^{1}\right) \widetilde{d}^{\dagger}\left(p^{1}\right)\right)},
$$

Notice that the Bogoliubov parameters are the same as before except for Fermi statistics

$$
\cos \theta_{F}(p ; \beta)=\frac{1}{\sqrt{1+e^{-\beta p}}},
$$

and that the Fermi-Dirac statistical weight is given by,

$$
N_{F}(p ; \beta)=\sin ^{2} \theta_{F}(p ; \beta)=\frac{1}{e^{\beta p}+1} .
$$

The thermal fermionic fields are straightforwardly introduced:

$$
\begin{aligned}
\psi\left(x^{ \pm} ; \beta\right) & =\int_{0}^{\infty} \frac{d p}{\sqrt{2 \pi}}\left\{f_{p}\left(x^{ \pm}\right)\left(b(\mp p) \cos \theta_{F}(p ; \beta)-\widetilde{b}^{\dagger}(\mp p) \sin \theta_{F}(p ; \beta)\right)\right. \\
& \left.+f_{p}^{*}\left(x^{ \pm}\right)\left(d^{\dagger}(\mp p) \cos \theta_{F}(p ; \beta)-\widetilde{d}(\mp p) \sin \theta_{F}(p ; \beta)\right)\right\} .
\end{aligned}
$$

and

$$
\begin{aligned}
\widetilde{\psi}\left(x^{ \pm} ; \beta\right) & =\int_{0}^{\infty} \frac{d p}{\sqrt{2 \pi}}\left\{f_{p}^{*}\left(x^{ \pm}\right)\left(\widetilde{b}(\mp p) \cos \theta_{F}(p ; \beta)+b^{\dagger}(\mp p) \sin \theta_{F}(p ; \beta)\right)\right. \\
& \left.+f_{p}\left(x^{ \pm}\right)\left(\widetilde{d^{\dagger}}(\mp p) \cos \theta_{F}(p ; \beta)+d(\mp p) \sin \theta_{F}(p ; \beta)\right)\right\} .
\end{aligned}
$$


The Fermion two-point function do not require regularizations:

$$
\begin{aligned}
\left\langle 0, \widetilde{0}\left|\psi\left(x^{ \pm} ; \beta\right) \psi^{\dagger}\left(y^{ \pm} ; \beta\right)\right| 0, \widetilde{0}\right\rangle & =\frac{1}{2 i \beta \sinh \left[\frac{\pi}{\beta}\left(x^{ \pm}-y^{ \pm}-i \varepsilon\right)\right]} ; \\
\left\langle 0, \widetilde{0}\left|\widetilde{\psi}\left(x^{ \pm} ; \beta\right) \widetilde{\psi}^{\dagger}\left(y^{ \pm} ; \beta\right)\right| 0, \widetilde{0}\right\rangle & =\frac{-1}{2 i \beta \sinh \left[\frac{\pi}{\beta}\left(x^{ \pm}-y^{ \pm}+i \varepsilon\right)\right]} ; \\
\left\langle 0, \widetilde{0}\left|\widetilde{\psi}\left(x^{ \pm} ; \beta\right) \psi\left(y^{ \pm} ; \beta\right)\right| 0, \widetilde{0}\right\rangle & =-\frac{1}{2 \beta \cosh \left[\frac{\pi}{\beta}\left(x^{ \pm}-y^{ \pm}\right)\right]} .
\end{aligned}
$$

This last correlator vanishes as $T \rightarrow 0$

Also notice that

$$
\left\langle 0, \widetilde{0}\left|\widetilde{\psi}\left(x^{ \pm} ; \beta\right) \psi^{\dagger}\left(y^{ \pm} ; \beta\right)\right| 0, \widetilde{0}\right\rangle=0
$$

\section{Free Massless Fermion thermofield Bosonization}

The bosonized expression for the Fermion fields are now defined as

$$
\begin{gathered}
\psi\left(x^{ \pm} ; \beta\right) \doteq W_{R}\left(x^{ \pm} ; \beta\right)=C \mathscr{Z}_{c}\left(\beta, \mu^{\prime}\right): e^{2 i \sqrt{\pi} \phi\left(x^{ \pm} ; \beta\right)}:, \\
\widetilde{\psi}\left(x^{ \pm} ; \beta\right) \doteq \widetilde{W}_{R}\left(x^{ \pm} ; \beta\right)=C \mathscr{Z}_{c}\left(\beta, \mu^{\prime}\right): e^{-2 i \sqrt{\pi} \widetilde{\phi}\left(x^{ \pm} ; \beta\right)}: .
\end{gathered}
$$

We stress that the anti commutations, with thermal Klein factors added, have been obtained. With the above expression the $\mathrm{N}$ point fermion functions are straightforwardly obtained with operatorial methods.

The symmetrized short distance fermionic currents turn out to be obtained as

$$
\mathscr{J}^{\mu}(x ; \beta)=: \bar{\psi}(x ; \beta) \gamma^{\mu} \psi(x ; \beta):=-\frac{1}{\sqrt{\pi}} \partial^{\mu} \phi(x ; \beta),
$$

thereupon the two point function is recovered

$$
\begin{array}{r}
\left\langle 0, \widetilde{0}\left|J^{ \pm}(x ; \beta) J^{ \pm}(y ; \beta)\right| 0, \widetilde{0}\right\rangle=\frac{1}{\pi} \partial_{x^{ \pm}} \partial_{y^{ \pm}}\langle 0, \widetilde{0}|\phi(x ; \beta) \phi(y ; \beta)| 0, \widetilde{0}\rangle \\
=\frac{-1}{4 \beta^{2}} \frac{1}{\sinh ^{2}\left[\frac{\pi}{\beta}\left(x^{ \pm}-y^{ \pm}-i \varepsilon\right)\right]} .
\end{array}
$$

The bosonization correspondence of the Lagrangians can be asserted: the short distance product of the free fermionic Hamiltonian leads to the free bosonic Hamiltonian. 


\section{Massless Thirring Model}

As an application of the bosonization formalism let us consider now the Thirring model at finite temperature:

$$
\mathscr{L}_{T}=i \bar{\psi} \gamma^{\mu} \partial_{\mu} \psi+\frac{1}{2} g\left(\bar{\psi} \gamma^{\mu} \psi\right)\left(\bar{\psi} \gamma_{\mu} \psi\right)-\left(-i \widetilde{\bar{\psi}} \gamma^{\mu} \partial_{\mu} \widetilde{\psi}+\frac{1}{2} g\left(\widetilde{\bar{\psi}} \gamma^{\mu} \widetilde{\psi}\right)\left(\widetilde{\bar{\psi}} \gamma_{\mu} \widetilde{\psi}\right)\right)
$$

A general finite temperature solution can be obtained as

$$
\psi(x)=\frac{1}{\sqrt{\pi}}(\mu)^{\frac{1}{4 \pi}\left(\alpha^{2}+\delta^{2}\right)}: e^{i\left(\alpha \gamma^{5} \varphi(x)+\delta \phi(x)\right)}:,
$$

where $\varphi$ is the Lorentz dual of $\phi$.

Indeed the thermalized $\mathrm{N}$-point functions result for the spin $1 / 2$ solution:

$$
\begin{gathered}
\left\langle 0, \widetilde{0}\left|\psi_{\alpha}\left(x_{1} ; \beta\right) \cdots \psi_{\alpha}\left(x_{n} ; \beta\right) \psi_{\alpha}^{\dagger}\left(y_{1} ; \beta\right) \cdots \psi_{\alpha}^{\dagger}\left(y_{n} ; \beta\right)\right| \widetilde{0}, 0\right\rangle=(2 \pi)^{-n}(i)^{-\frac{n}{2 \pi}\left(\delta^{2}+\frac{\pi}{\delta^{2}}\right)} \times \\
\prod_{i<j}^{n}\left[\frac{\beta}{\pi} \sinh \frac{\pi}{\beta}\left(x_{i}^{+}-x_{j}^{+}-i \varepsilon\right)\right]_{i, j}^{\frac{1}{4 \pi}\left(\delta^{2}+\frac{\pi^{2}}{\delta^{2}}+2 \pi \gamma_{\alpha \alpha}^{5}\right)} \prod^{n}\left[\frac{\beta}{\pi} \sinh \frac{\pi}{\beta}\left(x_{i}^{-}-x_{j}^{-}-i \varepsilon\right)\right]^{\frac{1}{4 \pi}\left(\delta^{2}+\frac{\pi^{2}}{\delta^{2}}-2 \pi \gamma_{\alpha \alpha}^{5}\right)} \times \\
\prod_{i<j}^{n}\left[\frac{\beta}{\pi} \sinh \frac{\pi}{\beta}\left(y_{i}^{+}-y_{j}^{+}-i \varepsilon\right)\right]^{\frac{1}{4 \pi}\left(\delta^{2}+\frac{\pi^{2}}{\delta^{2}}+2 \pi \gamma_{\alpha \alpha}^{5}\right)} \prod_{i, j}^{n}\left[\frac{\beta}{\pi} \sinh \frac{\pi}{\beta}\left(y_{i}^{-}-y_{j}^{-}-i \varepsilon\right)\right]^{\frac{1}{4 \pi}\left(\delta^{2}+\frac{\pi^{2}}{\delta^{2}}-2 \pi \gamma_{\alpha \alpha}^{5}\right)} \times \\
\prod_{i, j}^{n}\left[\frac{\beta}{\pi} \sinh \frac{\pi}{\beta}\left(x_{i}^{+}-y_{j}^{+}-i \varepsilon\right)\right]^{-\frac{1}{4 \pi}\left(\delta^{2}+\frac{\pi^{2}}{\delta^{2}}+2 \pi \gamma_{\alpha \alpha}^{5}\right)} \prod_{i, j}^{n}\left[\frac{\beta}{\pi} \sinh \frac{\pi}{\beta}\left(x_{i}^{-}-y_{j}^{-}-i \varepsilon\right)\right]^{-\frac{1}{4 \pi}\left(\delta^{2}+\frac{\pi^{2}}{\delta^{2}}-2 \pi \gamma_{\alpha \alpha}^{5}\right)} .
\end{gathered}
$$

\section{Conclusions}

Let us stress that the framework of thermofield Dynamics allows for a natural generalization of $T=0$ bosonization formulae in $1+1$ dimensions to the case of non-zero temperature. Also the "Mandelstam representation" for thermal fermions allows us to obtain in a compact way the exact and complete solution of a number of integrable models with massless fermions.

\section{Appendix}

Let us obtain the massless scalar field from the massive expressions. We start with the massive field 


$$
\begin{gathered}
\Sigma(x ; \beta)=U\left[\theta_{B}(\beta)\right] \Sigma(x) U\left[\theta_{B}(\beta)\right]= \\
\frac{1}{2 \sqrt{\pi}} \int_{-\infty}^{+\infty} \frac{d p}{\sqrt{p^{2}+m^{2}}}\left\{f_{p}(x)\left(a(p) \cosh \theta_{B}(p ; \beta)-\widetilde{a}^{\dagger}(p) \sinh \theta_{B}(p ; \beta)\right)\right. \\
\left.+f_{p}^{*}(x)\left(a^{\dagger}(p) \cosh \theta_{B}(p ; \beta)-\widetilde{a}(p) \sinh \theta_{B}(p ; \beta)\right)\right\} .
\end{gathered}
$$

The thermal two-point functions decomposes as:

$$
\langle 0, \widetilde{0}|\Sigma(x ; \beta) \Sigma(y ; \beta)| \widetilde{0}, 0\rangle=D_{o}^{(+)}(x-y ; m)+\mathscr{I}(x-y ; \beta, m),
$$

where $D_{o}^{(+)}(x-y ; m)=$ corresponds to zero temperature

$$
\begin{gathered}
D_{o}^{(+)}(x-y ; m)=\frac{1}{4 \pi} \int_{0}^{\infty} \frac{d p}{\sqrt{p^{2}+m^{2}}}\left\{e^{-i\left[\left(x^{0}-y^{0}\right) \sqrt{p^{2}+m^{2}}+\left(x^{1}-y^{1}\right) p\right]}+\right. \\
\left.e^{-i\left[\left(x^{0}-y^{0}\right) \sqrt{p^{2}+m^{2}}-\left(x^{1}-y^{1}\right) p\right]}\right\}
\end{gathered}
$$

and the temperature dependent part is:

$$
\begin{aligned}
\mathscr{I}(x-y ; \beta, m)= & \frac{1}{2 \pi} \int_{0}^{\infty} \frac{d p}{w} N_{B}(w, \beta)\left\{\cos \left[\left(x^{0}-y^{0}\right) w+\left(x^{1}-y^{1}\right) p\right]\right. \\
& \left.+\cos \left[\left(x^{0}-y^{0}\right) w-\left(x^{1}-y^{1}\right) p\right]\right\}
\end{aligned}
$$

with

$$
N_{B}(w, \beta)=\frac{1}{e^{\beta w}-1}=\frac{1}{e^{\beta \sqrt{p^{2}+m^{2}}}-1} .
$$

As long as $m \neq 0$ both integrals (A.3) (A.4) are regular.

Now introduce the infrared regulators $\mu$ and $\mu^{\prime}$ for each part and take $m \rightarrow 0$. Thus $N_{B}(w, \beta) \rightarrow$ $N_{B}(p ; \beta)$. It turns out that

$$
\begin{aligned}
D^{(+)}(x ; m, \beta)_{m \rightarrow 0}= & D_{o}^{(+)}(x)+\frac{1}{2 \pi} \int_{\mu^{\prime}}^{\infty} \frac{d p}{p} N_{B}(p ; \beta)\left[\cos p\left(x^{+}\right)+\right. \\
& \left.\cos p\left(x^{-}\right)\right]
\end{aligned}
$$

This leads naturally to the splitting into chiral two-point functions:

$$
\left\langle 0, \widetilde{0}\left|\phi\left(x^{ \pm} ; \beta\right) \phi\left(0^{ \pm} ; \beta\right)\right| \widetilde{0}, 0\right\rangle=D_{o}^{(+)}\left(x^{ \pm}\right)+\frac{1}{2 \pi} \int_{\mu^{\prime}}^{\infty} \frac{d p}{p}\left[\cos p\left(x^{ \pm}\right)\right] N_{B}(p ; \beta),
$$

with

$$
D_{o}^{(+)}\left(x^{ \pm}-y^{ \pm}\right)=-\frac{1}{4 \pi} \ln \left[i \mu\left(x^{ \pm}-y^{ \pm}-i \varepsilon\right)\right]
$$


With this the thermalization of the chiral components is justified. Within this procedure $\mu^{\prime}$ and $\mu$ should indeed be identified. Both came out from infrared regularization in (A.3) and (A.4).

\section{References}

[1] E. Abdalla, M.C.B. Abdalla and K.D. Rothe, "Non-Perturbative Methods in two dimensional Quantum Field Theory", World Scientific, Singapore, 1991, and idem. 2nd edition, 2001.

[2] D. Delepine, R. Gonzales Felipe and J. Weyers, Phys. Lett. 419 (1998) 296 ; A. Gomez Nicola and D.A. Steer, Nucl. Phys. B549 (1998) 409; A. Gomez Nicola, R.J. Rivers and D.A. Steer, Nucl. Phys. B570 (2000) 475.

[3] A. Liguori, M. Mintchev, L. Pilo, Nucl. Phys. 569 (2000)577;

[4] H. Umezawa, H. Matsumoto, M. Tachiki, "Thermo Field Dynamics and Condensed States", Nort-Holland 1982;

[5] I. Ojima, Ann. phys. 137 (1981) 1;

[6] H. Matsumoto, I. Ojima and H. Umezawa, Ann. Phys. 152 (1984) 348.

[7] R. L. P. G. Amaral, L. V. Belvedere and K. D. Rothe, Ann. Phys 320 (2005) 399. 